Volume 2, Issue 2, April-June 2017, Pages: 164, DOI: http://dx.doi.org/10.19082/ah164

\title{
CREATING THE FIRST REGISTRY FRAMEWORK FOR COCHLEAR IMPLANTED PATIENTS IN IRAN: A PRELIMINARY REPORT
}

\author{
Arash Bayat ${ }^{1}$, Nader Saki ${ }^{2}$, Soheila Nikakhlagh ${ }^{3}$, Majid Karimi ${ }^{4}$, Emel Saki Malehi ${ }^{5}$, Golshan Mirmomeni ${ }^{6}$, \\ Mohammad Nikafrooz ${ }^{7}$
}

1: Hearing Research Center, Ahvaz Jundishapur University of Medical Sciences, Ahvaz, Iran

2: Hearing Research Center, Ahvaz Jundishapur University of Medical Sciences, Ahvaz, Iran

3: Hearing Research Center, Ahvaz Jundishapur University of Medical Sciences, Ahvaz, Iran

4: Department of Audiology, Iran University of Medical Sciences, Tehran, Iran

5: Department of Biostatistics, Ahvaz Jundishapur University of Medical Sciences, Ahvaz, Iran

6: Student Research Committee, Ahvaz Jundishapur University of Medical Sciences, Ahvaz, Iran

6: MA in TEFL, Ahvaz Branch, Payam Noor University, Ahvaz, Iran

\section{TYPE OF ARTICLE: CONFERENCE ABSTRACT}

\begin{abstract}
Introduction: Currently, there is a paucity of data concerning the long-term outcomes, educational placement and quality of life of cochlear implanted (CI) patients in Iran. Therefore, we decided to design a national CI registry to facilitate communication and collaboration, improve care, and create a framework for aggregate data sharing in CI research.

Methods: A group of nationally represented, multidisciplinary CI providers cooperated to define a standard set of data elements to incorporate into a database built by them in association with a group of computer scientists and software designers. The CI registry database has been designed so that different centers across the country will be able to use the database for their own clinical purposes. The database utilizes a secure web interface to administer electronic case report forms to clinicians and families of implanted children. Patients will be evaluated with a set of standardized questionnaires prior to initial device activation (baseline) and at three-monthly follow-up intervals up to 24 months and annually thereafter.

Results: Approximately 6 months after the first release of the database, clinical information on 123 patients has been entered from Khuzestan CI center. The information regarding to patient comorbidities, device use, auditory performance, quality of life and health-related utilities, across different types of CI devices were recodeded in the database.

Conclusion: A standardized CI registry database that is utilized by a growing network of CI centers can help strengthen research through aggregate data sharing.
\end{abstract}

KEYWORDS: Registry, Cochlear, Iran

\footnotetext{
Abstracts of First National Congress of Medical Informatics, Mashhad, Iran, February 2017

(C) 2017 The Authors. This is an open access article under the terms of the Creative Commons Attribution-NonCommercialNoDerivs License, which permits use and distribution in any medium, provided the original work is properly cited, the use is non-commercial and no modifications or adaptations are made.
} 